\title{
OPTICAL MODULATION EFFECTS IN INDIUM OXIDE FILMS
}

\author{
J. AVARITSIOTIS and R. P. HOWSON \\ Department of Electrical and Electronic Engineering, University of Technology, Loughborough, \\ Leicestershire, U.K.
}

(Received May 28, 1976; in final form April 5, 1977)

\begin{abstract}
Tin doped indium oxide films produced by chemical vapour deposition and by d.c. sputtering have shown an optical reflectance spectra which can be modulated in the region of the plasma edge in the near infra-red by an electric field applied to the surface through an electrolyte. The effect produced could be quantitatively predicted by a theory developed for surfaces whose optical properties were controlled by their free carrier properties and for the sample being in thin film form.

The plasma edge for films prepared by chemical vapour deposition on some occasions showed a modified plasma edge effect which was attributed to surface plasmons allowed by roughness effects.
\end{abstract}

\section{INTRODUCTION}

Tin doped indium oxide films arouse interest in that they can be produced in an electrically conducting form whilst maintaining optical transparency to visible radiation. They have found application in the radiation insulation of sodium lamps ${ }^{1}$ and domestic windows, ${ }^{2}$ which leads to their exploitation in solar energy converters, electro-optic devices ${ }^{3}$ and thin film optical wave-guides. ${ }^{4}$ They have provided very convenient electrodes for electro-chemical experiments where effects are being studied which occur close to the surface of the electrode. ${ }^{5}$ The effect of the applied electric fields on the optical properties of the films has not been studied.

These films have optical properties which, for the spectral region where the transition from an optically transparent to an opaque highly reflecting state occurs, depend upon the properties of the free carriers independent of electron transitions between allowed energy bands which are far removed from these wavelengths. This offers a system where the validity of recent quantitative theories applicable to conducting surfaces and to thin film samples can be tested. Previous measurements have been made on gold and silver where there is considerable interference from inter-band effects. ${ }^{6}$ The optical properties of the film in the transition region are of considerable interest in any optical application in that the limit of the range of transparency and the limit of the range of high reflectivity and less emissivity are defined.

\section{EXPERIMENTAL DETAILS}

Films were used that had been prepared by chemical vapour deposition (C.V.D.) and by d.c. sputtering; both on to glass substrates. The C.V.D. films were prepared by the pyrolytic decomposition of indium and stannic chloride in methyl alcohol on a substrate heated to about $500^{\circ} \mathrm{C}$, at a rate of about $200 \mathrm{~nm} \mathrm{~s}^{-1}$. Sputtered films were made from prepared sources by D.C. sputtering of conducting mixed compacted source oxides onto glass substrates at a pressure of $10^{-4}$ torr at room temperature. The film thicknesses were in the range $26 \mathrm{~nm}$ to $59 \mathrm{~nm}$ and were measured from the thickness interference fringes shown as a function of wavelength in the visible region of the spectrum where the films were transparent.

The free carrier concentration of Sn doped films of $\mathrm{In}_{2} \mathrm{O}_{3}$ films is shown to vary between approximately 0.3 to $1.5 \times 10^{21} \mathrm{~cm}^{-3}$ dependent upon the number of oxygen vacancies introduced during the fabrication process. ${ }^{8}$ These densities give plasma wavelengths in the near infra-red. Optical measurements in the region of most interest, the near infrared, were made by point to point plotting of normal incidence reflectance in comparison to that of a freshly prepared aluminium coated mirror. A double grating monochromator was used with a Golay detector and phase sensitive processing techniques. For the visible region of the spectrum a double beam recording spectrophotometer (Unicam SP 5000) was used. Inspection of the surfaces of films of similar thickness with a scanning electron microscope showed 
that in general the films prepared by sputtering were considerably smoother with graduated variations of the surface with a periodicity of about $250 \mathrm{~nm}$ compared to much sharper variations at a period of about $100 \mathrm{~nm}$ for those prepared by C.V.D.

An electric-field was applied to the surface of the oxide film through the double layer of an electrolytic cell. The electrolyte, chosen after experimental investigation, was aceto-nitrile which gave a large double layer capacitance $\left(0.16 \mathrm{~F} / \mathrm{m}^{2}\right)$ such that a large electric field could be applied to the surface but had some absorption bands in the spectral region of interest which limited the measurements. The electrolytic cell incorporated a sodium chloride window through which the radiation passed onto the sample, and a large surface area platinum mesh counter electrode. This system allowed that modulation voltages of up to $20 \mathrm{~V}$ to be applied without any evidence of electro-chemical activity and degradation of the reflectivity of the sample. Phase sensitive detection techniques, with the modulating voltage providing the reference, enabled sufficient sensitivity to be obtained for measurement of the relatively small electro-reflectance effect.

\section{THEORY}

The position and sharpness of the plasma edge shown by the films is dependent upon the preparation of the films, as is the electrical conductivity, through the free carrier density, $N$, and the relaxation time, $\tau$.

The description of the optical properties around the plasma edge is given by the theory of Drude, i.e.

$$
\epsilon=\epsilon_{L}\left(1-\frac{\omega_{p}^{2}}{\omega^{2}+j \omega \tau^{-1}}\right)
$$

where $\epsilon$ is the dielectric constant of the material and $\epsilon_{L}$ that of the lattice alone. $\omega_{p}$ is the plasma frequency given by

$$
\omega_{p}^{2}=N e^{2} / m^{*} \epsilon_{0} \epsilon_{L},
$$

$m^{*}$ is the effective mass of the free carriers. The reflectance, $R$, is given by

$$
R=\frac{\left(n_{0}-n\right)^{2}+k^{2}}{\left(n_{0}+n\right)^{2}+k^{2}}
$$

where $\epsilon=(n-j k)^{2}, n$ is the refractive index and $k$ the extinction coefficient. With some materials it is possible to observe a kink in the plasma edge which is attributed to an additional mode of resonance due to surface plasmons allowed by surface roughness; the frequency is given from: ${ }^{7}$

$$
\epsilon_{e}\left(\omega_{s}\right)=-\epsilon\left(\omega_{s}\right)
$$

where $\epsilon_{e}$ is the dielectric constant of the electrolyte, the other material at the interface. When $\omega \tau \gg 1$ then the Drude model gives

$$
\omega_{s}=\frac{\omega_{p}}{\sqrt{\left(\epsilon_{e} / \epsilon_{L}\right)+1}}
$$

The normalised changes in reflectance due to an electric field at the surface of a thin film have been given as: ${ }^{6}$

$$
\frac{\Delta R}{R V}_{E R S}=\left(W_{1}-W_{2}\right) \cdot\left[\frac{\Delta R_{1}}{R_{1} \cdot V}\right]
$$

where $R$ and $\Delta R$ refer to the reflectance of the thin film system in terms of the surface effect $R_{1}$.

and

$$
\begin{aligned}
& 1+\left(R_{2}^{1 / 2} / R_{1}^{1 / 2}\right) \exp (-\beta) \cos \phi+ \\
& W_{1}=\frac{+2 H\left(R_{2}^{1 / 2} / R_{1}^{1 / 2}\right) \exp (-\beta) \sin \phi}{1+\left(R_{2} / R_{1}\right) \exp (-2 \beta)+} \\
& +2\left(R_{2}^{1 / 2} / R_{1}^{1 / 2}\right) \exp (-\beta) \cos \phi
\end{aligned}
$$

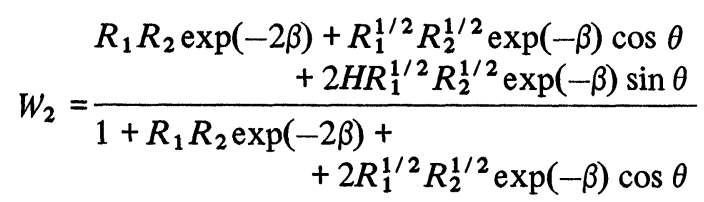

$$
H=\frac{\omega \tau\left(n^{2}-k^{2}-n_{0}^{2}\right)+2 n k}{n^{2}-k^{2}-n_{0}^{2}+2 n k \omega \tau}
$$

$\beta=(2 \omega d / c) k, \phi$ and $\theta$ are overall phase shifts, ${ }^{6} d$ is the film thickness, $R_{1}=\left|r_{1}\right|^{2}, R_{2}=\left|r_{2}\right|^{2} ; r_{1}$, refers to the interface between electrolyte and film material and $r_{2}$ to that of the film and the substrate. Finally,

$\left[\frac{\Delta R_{1}}{R_{1} V}\right]=\frac{4 n_{0} q \tau}{c m^{*} \epsilon_{0}} \cdot\left[\frac{-\epsilon_{A}+\epsilon_{1}+\epsilon_{B} \omega \tau}{\left(\omega^{2} \tau^{2}+1\right) \cdot\left[\left(\epsilon_{A}-\epsilon_{e}\right)^{2}+\epsilon_{B}^{2}\right]}\right]$

where: $\epsilon_{A}=n^{2}-k^{2}, \epsilon_{B}=2 n k, \epsilon_{e}=n_{0}^{2}, n_{0}$ is the refractive index of the electrolyte and $n_{1}$ that of the substrate. 
$C$ is the capacitance of the configuration used for the application of the low frequency electric field on the surface of the film (i.e., interface 1).

The important role of $m^{*}, \tau, \epsilon_{L}$ in the spectral distribution of the effect is evident; a large electroreflectance effect is expected in materials where the free carrier mobility, $\mu=\left(\tau / m^{*} q\right)$ is large and $\epsilon_{L}$ is small. This in terms of reflectivity implies that a sharp reflectance plasma edge and a low value for the reflectivity minimum are the necessary features of a material to yield large reflectance modulation percentages. The density of the free carriers determines the wavelength at which the plasma edge appears.

\section{RESULTS}

Figure 1 shows the plasma edge of two films prepared by C.V.D. and illustrates the kink in the edge which is attributed to surface roughness and has previously been observed by Kostlin. ${ }^{1}$ In general it is possible to say that these films prepared by C.V.D. gave sharper plasma edges i.e. higher values of $\tau$ and therefore free carrier mobility than those sputtered and this is attributed to the greater structural order given by the higher substrate temperatures that were used.

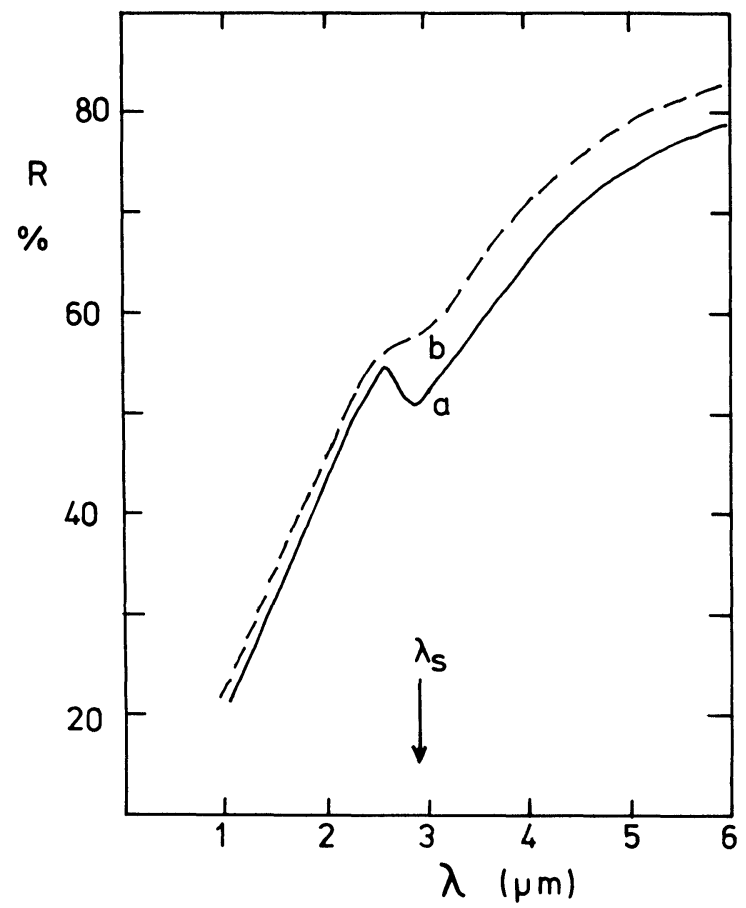

FIGURE 1 The reflectivity spectra of two $\mathrm{Sn}$ doped $\mathrm{In}_{2} \mathrm{O}_{3}$ films prepared by C.V.D. showing the effect of surface plasmons.

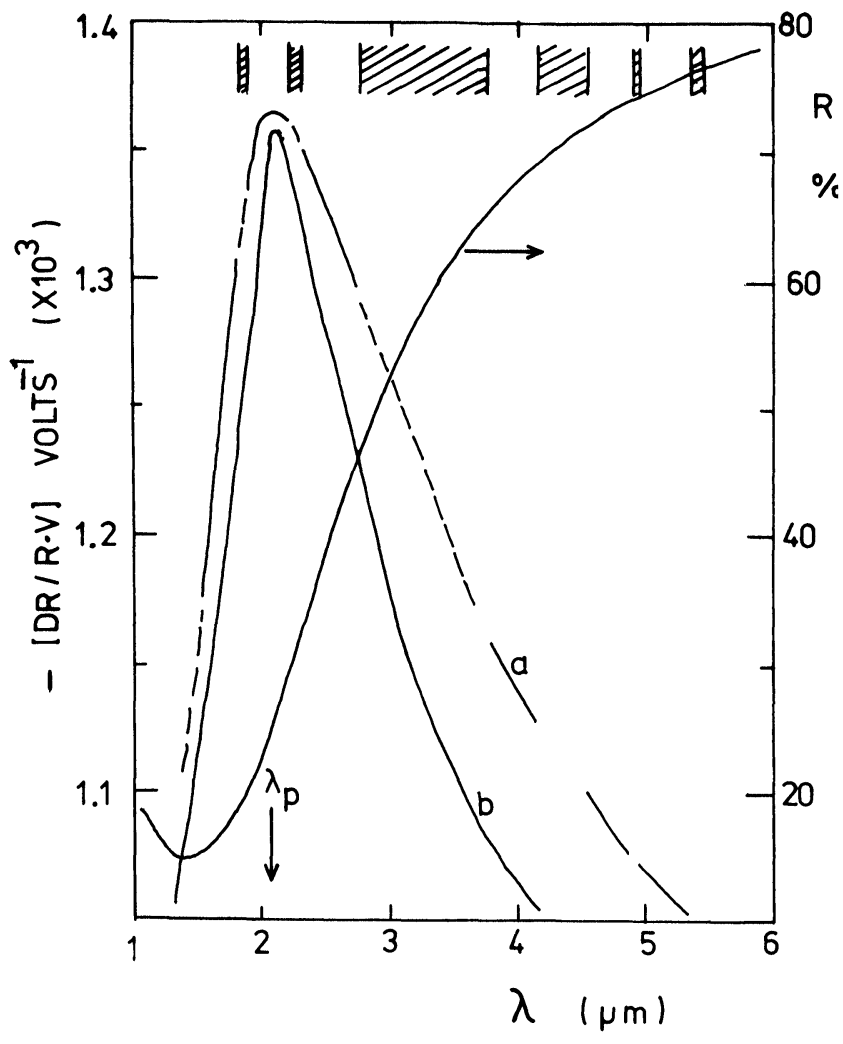

FIGURE 2 (a) Experimental and (b) calculated electroreflectance spectra for a $\mathrm{Sn}$ doped $\mathrm{In}_{2} \mathrm{O}_{3}$ film of $27 \mathrm{~nm}$ thickness prepared by C.V.D. Also shown is the reflectance spectra and the absorption bands in the aceto-nitrile electrolyte which prevented measurements being made. (Electrolytic capacitance, $C$, was $10 \mu \mathrm{Fm}^{-2}$ ).

Figures 2 and 3 show the effects observed with a film prepared by C.V.D. and d.c. sputtering respectively; the reflectance and the experimental and theoretical electro-reflectance are shown as a function of wavelength. The theoretical effect is obtained by analysing the experimental plasma reflectance edge, and adjusting the parameters in a computer programme giving the reflectance of a thin film on a substrate affected only by free carrier and dielectric optical effects until a good match was obtained. ${ }^{10}$ This gives the optical constants of the material in that region of the spectrum. This could then be used to substitute in the theoretical formula, Eq. (5), to predict the electroreflectance effect.

It can be seen that very good agreement is obtained both in the estimation of the magnitude of the effect and in its spectral distribution especially considering the approximation and assumptions 


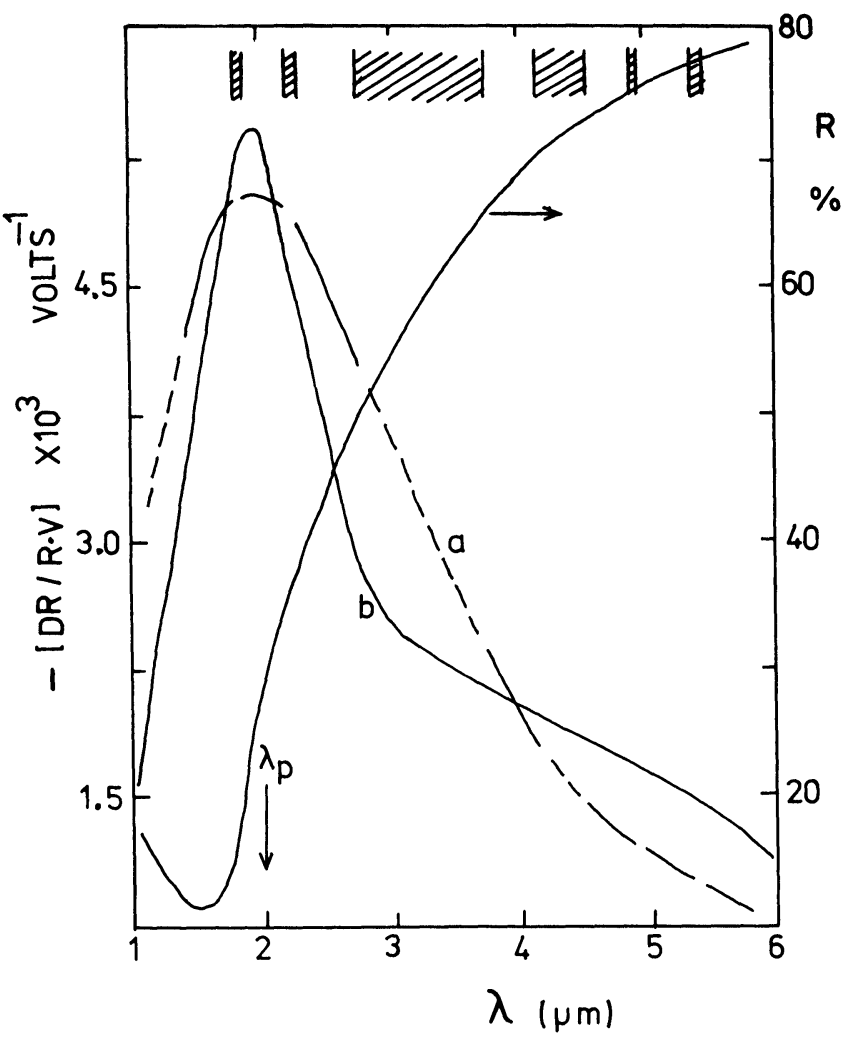

FIGURE 3 (a) Experimental and (b) calculated electroreflectance spectra and absolute reflectance spectra for a $\mathrm{Sn}$ doped $\mathrm{In}_{2} \mathrm{O}_{3}$ film of $45 \mathrm{~nm}$ prepared by d.c. sputtering. (Electrolytic capacitance was $16 \mu \mathrm{Fm}^{-2}$ ).

inherent in a macroscopic theory dealing with a thin layer of enhanced carrier of atomic dimensions. The electro-reflectance of other samples with different free carrier effects, which are demonstrated by the position and shape of the plasma edge, may be confidently predicted.

\section{CONCLUSIONS}

An electro-reflectance effect has been demonstrated in thin films of $\mathrm{Sn}$ doped $\mathrm{In}_{2} \mathrm{O}_{3}$ which depends for its magnitude on the sharpness of the plasma reflectance edge and through that on the properties of the free carriers, their relaxation time and effective mass and hence their mobility. It has been shown that the experimental electro-reflectance effect can be predicted quantitatively by a theory based on enhanced density of free carriers produced in a very thin surface layer by the applied electric field.
The specific values of electro-reflectance obtained will depend on the reflectance edge shown by the film and this in its turn will depend on preparation conditions. However, these conditions have not been specified in detail as it has been shown that the electro-reflectance can be predicted over a wide range of reflectance edge values.

The conclusions drawn from the work reported here allow us to predict that for films of $\mathrm{In}_{2} \mathrm{O}_{3}$ which give higher relaxational mobilities, larger changes of the reflectance induced by an electric field will be obtained and can be used as a measure of the sharpness of the plasma edge as well as offering a wavelength tuned optical modulator. Films of $\mathrm{Zr}$ doped $\operatorname{In}_{2} \mathrm{O}_{3}$ have been reported with mobilities considerably higher $\left(170 \mathrm{~cm}^{2} V^{-1} \mathrm{~s}^{-1}\right)^{11}$ than those used here $\left(10 \mathrm{~cm}^{2} V^{-1} \mathrm{~s}^{-1}\right)$ and would be expected to have considerably greater electro-reflectance effects.

That films of $\operatorname{In}_{2} \mathrm{O}_{3}$ have been shown to withstand up to 20 volts applied across a double layer in an electrolytic cell with no evidence of surface activity suggests a suitable system for electrochemical investigation of absorbed species and the kinetics of fast homogeneous reactions involving electro-generated species. Internal or external reflection spectroscopy could be used depending upon the transparency of the electrolyte solution.

\section{ACKNOWLEDGEMENTS}

We would like to thank Thorn Co. Ltd., and Plessey Co. Ltd., who kindly provided samples of Sn doped in $\operatorname{In}_{2} \mathrm{O}_{3}$ and to SRC who supported this work.

\section{REFERENCES}

1. H. Kostlin, Philips Tech. Rev., 34, 242 (1974).

2. H. J. J. van Boort and R. Groth, Philips Tech. Rev.. 29, 17 (1968).

3. R. Clanget, Appl. Phys., 2, 247 (1973).

4. D. B. Fraser and H. D. Cook, J. Electro. Chem. Soc., 119, 1368 (1972).

5. W. N. Hansen, Advances in Electro. Chem. and Electro. Chem. Eng., 9, 50 (1973).

6. R. P. Howson, J. N. Avaritsiotis and T. Fox, Thin Solid Films, 30, 297 (1975).

7. J. N. Avaritsiotis and R. P. Howson. To be published.

8. H. Kostlin, R. Just and W. Lems, Phys. Stat. Sol., 29, No. 1, 89 (1975).

9. E. A. Stern and R. A. Ferrell, Phys. Rev., 120,130 (1960).

10. J. N. Avaritsiotis and R. P. Howson. To be published.

11. R. Groth, Phys. Stat. Sol., 14, 69 (1966). 

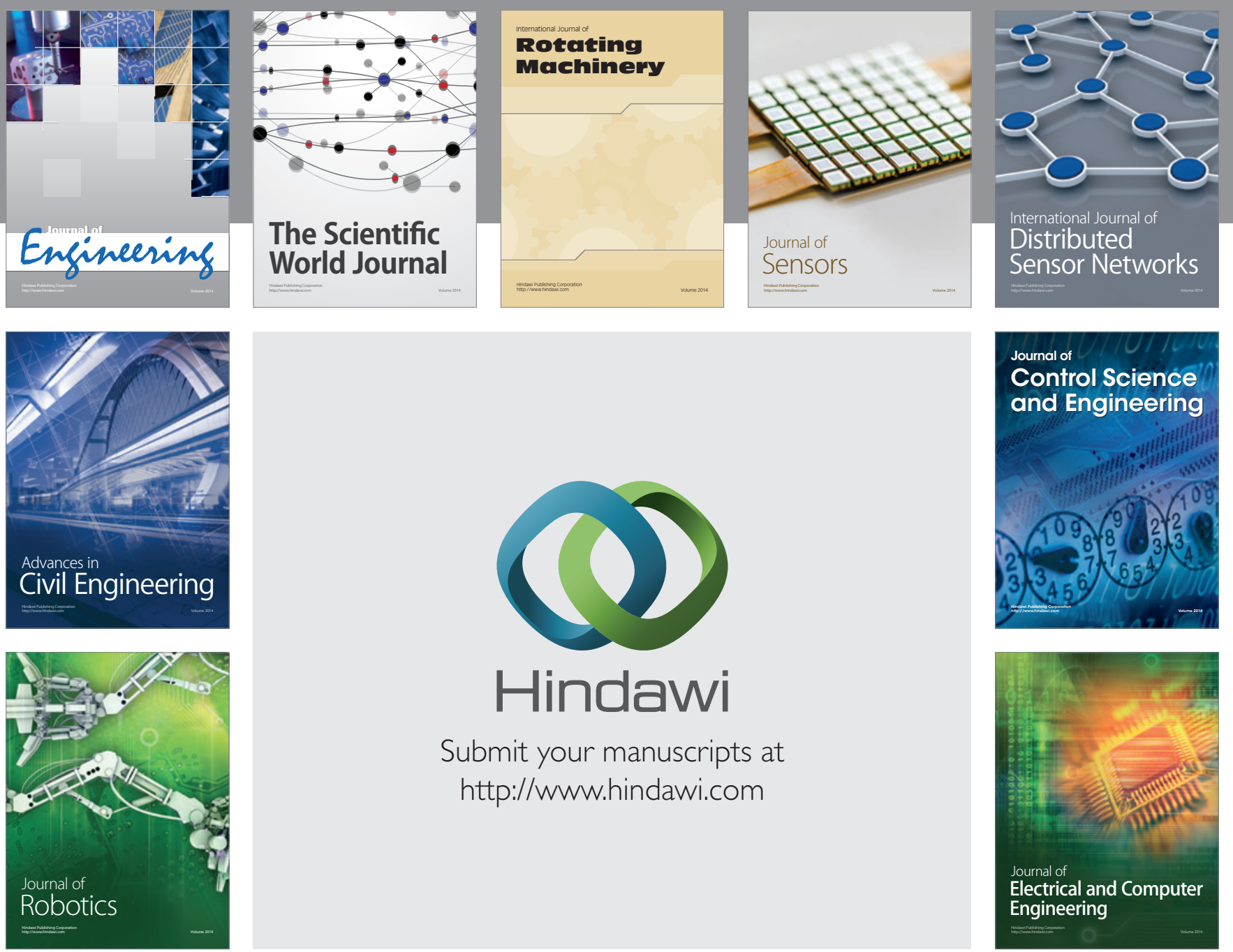

Submit your manuscripts at

http://www.hindawi.com
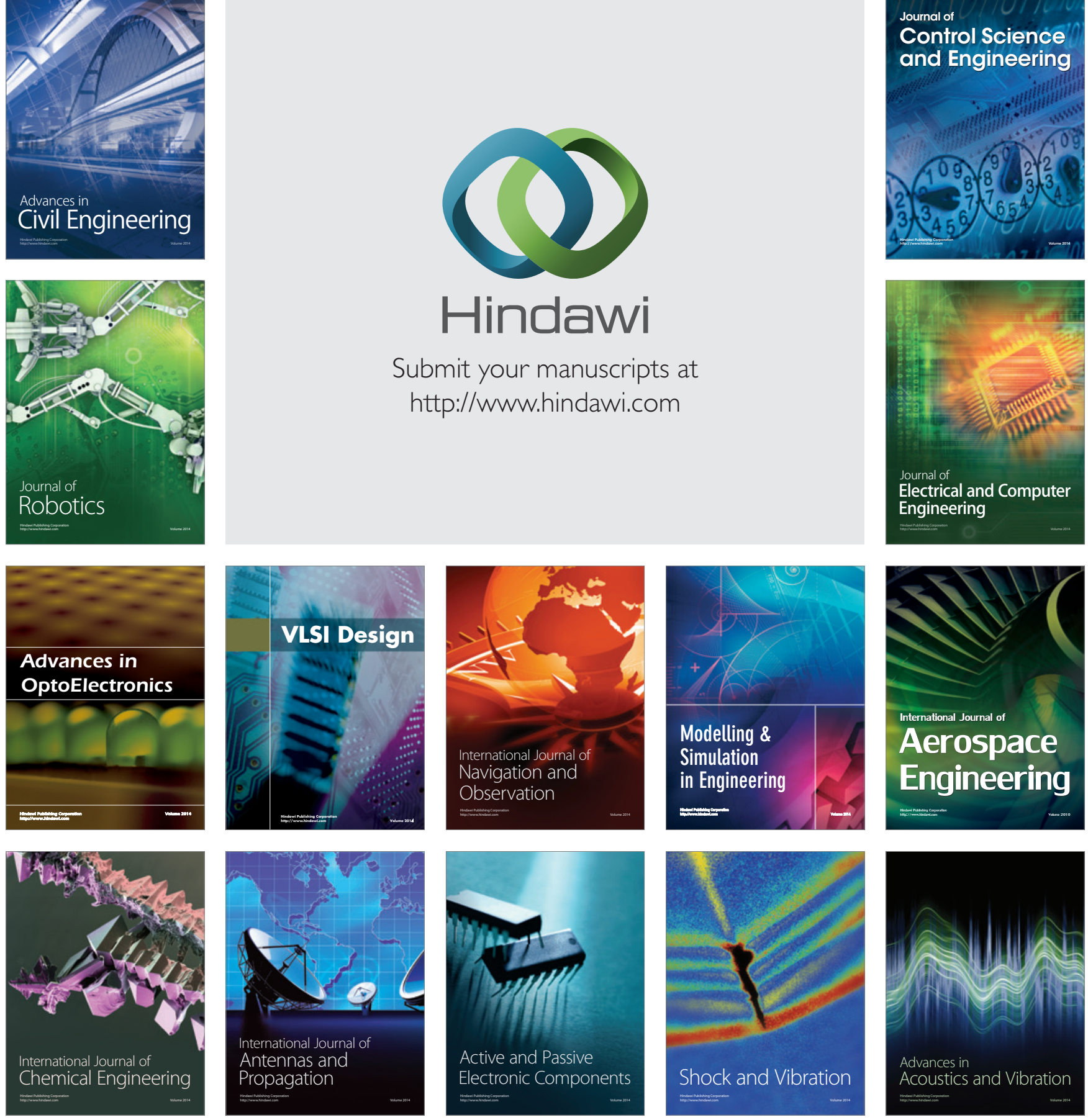